- PPPL 1886

UC20f

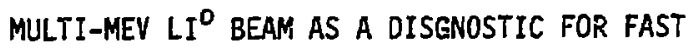

CONFINED ALPHA PARTICLES

BY

L.R. Grisham, D.E. Post, and D.R. Mikkelsen

April 1982
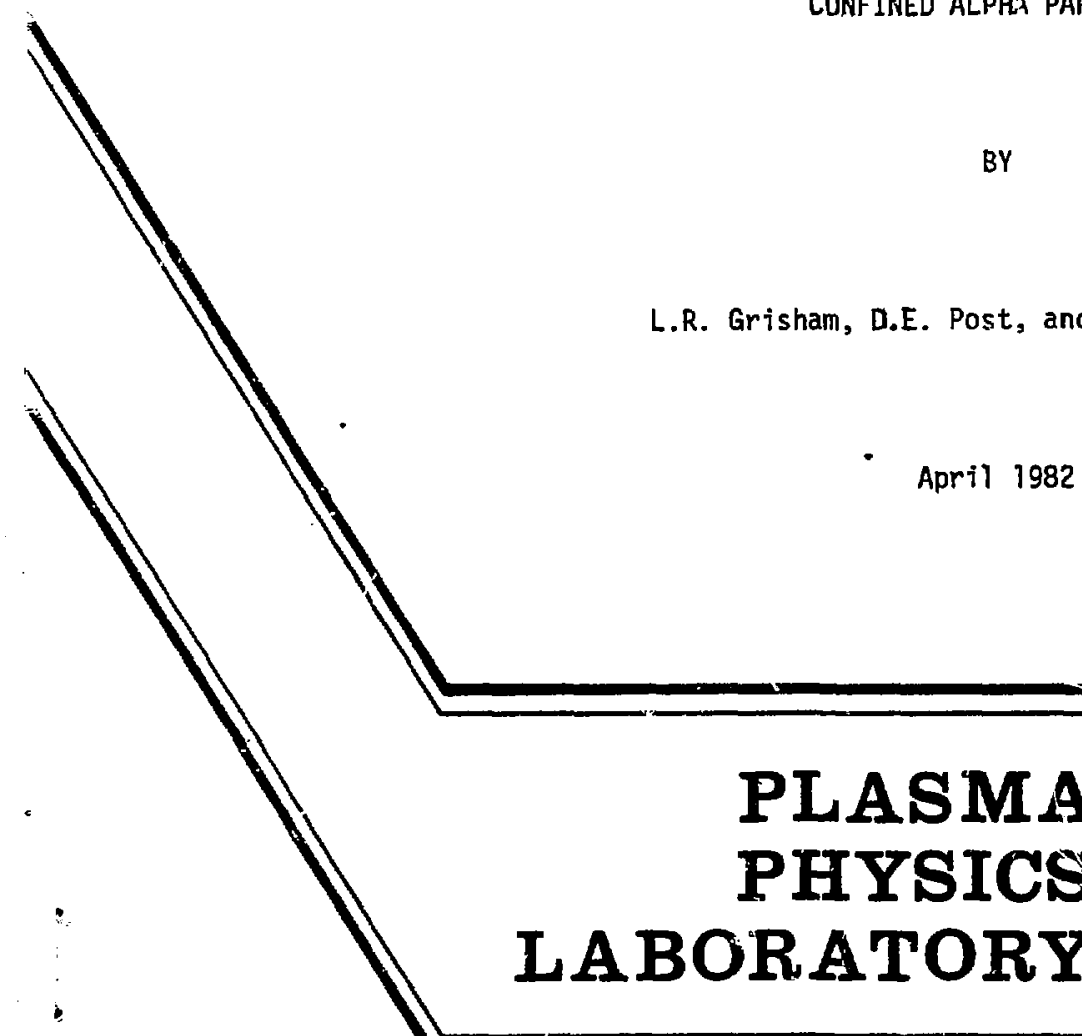

(

r

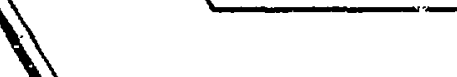

PLASMA

PHYSICS

LABORATORY

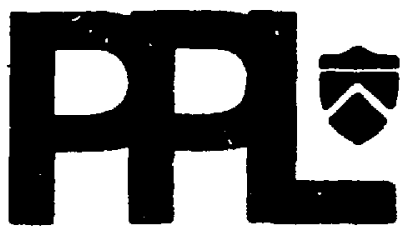

PRINCETON UNIVERSITY

PRINCETON, NEW JERSEY

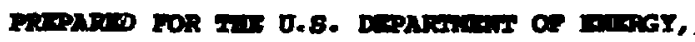

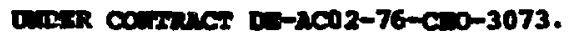


PPPL- -1886

DE\& 2009821

\title{
Multi-MeV $\mathrm{Li}^{\circ}$ Beam as a Diagnostic for Fast Confined Alpha Particles
}

\author{
L. R. Grisham, D. E. Post, D. R. Mikkelser \\ Princeton University Flasma Physics Laboratory \\ P. O. Box 45I, Princeton, N.J. 08544
}

\begin{abstract}
We discuss a method of measuring the velocity distribution of crnfined energetic alpha particles resulting from deuteriumtritium fusion reactions in a magnetically contained plasma. We calculate the characteristics of the signals to be expected from injecting multi-MeV Li ${ }^{\circ}$ into the plasma to undergo double chargeexchange reactions with the alpha particles. Neutralized alpha particles then escape from the plasma to be detected by a chargeexchange analyzer, We also examine the feasibility of producing a $\mathrm{Li}^{\circ}$ beam of the required current and energy, and we discuss a conceptual design for an appropriate beam system.
\end{abstract}




\section{Intraduction}

Many magnetic confinement fusion reactor concepts rely upon substantial heating of the plasma by the D-T fusion-product alpha particles as they slow down classically. However, a number of authors [1] have studied anomalous processes which might alter the slowing down of the alpha particles, thereby changing their energy transfer to the plasma. In gereral, these anomalous processes could lead to loss of the fast alphas from the central portion of the plasma, or they could change the rate at which the fast alpha particles slow down and heat the ions and electrons. The anomalous loss of alpha particles from the central portion of a plasma being heated to ignition could increase the $n \tau_{E}$. auxiliary heating power, and $\beta$ required for ignition. Anomalous ion heatiry by alpha particles could lead to a reduction in ignition requirements. In addition, the loss of alpha particles from the whole plasma could result in accelerated erosion of the reactor first wall from blistering $[2,3]$ due to bombardment by the escaping energetic alpha particles. Given the large costs and lead times associated with reactor-sized experiments, it is desirable to discover at the earliest possible date whether the fusion-product alpha particles slow down in a classical manner through binary coulomb collisions, or whether instead they are subject to anor $>$ lous processes prior to thermalization. The first generation of $\mathrm{D}-\mathrm{T}$ burning tokamaks is unlikely to provide answer about alpha confinement through the power balance alone. At $Q=1$ alpha particle heating will account for only about a fifth of the input power to the plasma, even if the alpha particles thermalize compietely 
before they are lost. Consequently, a diagnostic is needed to measure the slowing down spectrum of the confined alpha particles in much the same way that charge-exchange analyzers have measured the slowing down spectrum of beam-injected ions by looking at the charge-exchange flux [4].

In the normal course of events, however, the fast confined alpha particles should have little chance for double charge exchange in the hot core of a $Q=1$ plasma. Recently, Post et. al. [5] proposed that one might locally enhance the charge-exchange probability of the alpha particles by injecting a neutral doping beam. This beam would undergo single or double charge-exchange events with fast alpha particles. One might observe either the doppler-shifted de-excitation radiation from the decay cf excited $\mathrm{He}^{+}$states populated by single charge excinange, or the fast $\mathrm{He}^{\mathrm{o}}$. escaping from the plasma after acquiring two electrons through double charge exchange with the beams.

The charge-exchange cross sections for $\mathrm{He}^{++}$decline rapidly at relative velocities above $\sim 4 \times 10^{8} \mathrm{~cm} / \mathrm{sec}$ becalse the orbital velocities of the bound electrons in Helium are of this order. Since the D-T fusion-product alpha particles are born at $\sim 1.3 \times 10^{9} \mathrm{~cm} / \mathrm{sec}$, the doping beam must be capable of velocities about as high as the birth velocity (corresponding to $880 \mathrm{kev} / \mathrm{amu}$ ) in order to have a significant probability of charge exchange. From this velocity requirement it follows that tile successiui development of this diagnostic method will pose significant technical difficulties.

This paper relates the results of a study we have done of 
one approach to the alpha particle diagnostic: double charge exchange from a doping beam, followed by analysis of the velocity distribution of the resulting $\mathrm{He}^{\mathrm{O}}$ which escapes. We compute the expected signals, and we discuss a possible design for the diagnostir. We have chosen to use double charge exchange because the background problem should be much less severe than would be the case in looking at the doppler-shifted radiation following single charge exchange. The signal from the latter process would be about the same intensity as bremsstrahlung, which is itself expected to constitute only a small fraction of the background near $300 \AA$.

The present paper extends the work of Ref. 5. in that it studies the use of a specific reaction, and explores the appropriate beam technology required, for an alpha diagnostic based upon the atomic processes discussed in Ref. 5.

II. Choice of a Doping Beam

The requirement that the beam energy should be able to reach as high as $\sim 880 \mathrm{keV} / \mathrm{amu}$ mandates the use of negative ions as precursors to a neutral beam, regardless of the beain used. In order to minimize the cost, one would prefer to use the lightest doping atom possible, since this would result in the lowest beam energy requirement. Accordingly, the most obvious candidate for a double charge exchange doping beam is ${ }^{3} \mathrm{He}$, for which the negative ions can be made in adequate quantity (10's of $\mathrm{mA}][6,7]$. Unfortunately the $\mathrm{He}^{\circ}$ resulting from neutralization of $\mathrm{He}^{-}$will be predominately in a long-lived metastable state, $\left[\mathrm{He}^{+}\left(1 \mathrm{~s} 2 \mathrm{p}^{3} \mathrm{~s}\right)\right][8]$. It would be extremely difficult to determine 
either the double charge-exchange cross sectson for the metastable component of the beam or the percentage of the beam in the ground state. Further, the metastable portion of the beam could not be quenched without ionizing most of it [9].

These considerations lead one to choose the next lightest atom, ${ }^{6} \mathrm{Li}$, as the probe beam. The beam resulting from neutralization of ${ }^{6} \mathrm{Li}^{-}$should be primarily in the ground state or in a state which will rapidly decay to the ground state.

III. Signal Computation

At the time of Ref. 5, the cross section for the reaction $\mathrm{Li}^{\circ}+\mathrm{He}^{++} \longrightarrow \mathrm{Li}^{++}+\mathrm{He}^{\circ}$ was not known. Since then two groups have measured this cross section as a function of energy $[10,11]$, obtaining good agreement with each other. Figure 1 shows the cross-section curve used in these calculations. This is a composite of the measurements by Roferences 10 and 11 . The curve has been extrapolated at energies below $28 \mathrm{keV}$ and above $800 \mathrm{keV}$. We have used the cross section in Fig. 1 to calculate the characteristics of the $\mathrm{He}^{\circ}$ flux resulting from double charge exchange. We have done these calculations for two assumed alpha particle velocity distribution functions. The first, which we shall refer to as the "classical distribution," is that which would result from slowing down of the alpha particles entirely by classical binary collisions on a TFTR plasma [5] with $T_{e}=10 \mathrm{keV}, \mathrm{T}_{i}=20 \mathrm{keV}$, and $\mathrm{n}_{e}=1 \times 10^{14} \mathrm{~cm}^{-3}$. The other ail-tribution, which we shall refer to as "nonclassical," is the classical distribution multiplied by a $\left(v / v_{0}\right)^{4}$ term, where $v_{0}$ is the birth velocity of the fusion-product alpha particles. 
Such a distribution might result from a process which caused rapid loss of the fast alpha particles. Comparing the results arising from these two distributions gives us an indication of the sensitivity of the emerging flux to gross variations in the alpha particle containment.

Figure 2 shows $\langle\sigma \mathrm{V}\rangle$ as a function of the $\mathrm{Li}^{\circ}$ doping beam velocity for the classical (a) and the assuned nonclassical distribution (b). Thus, if one put in a doping beam at successively different velocities, one would observe very different count rates and (below $v_{\text {beam }}=10^{9} \mathrm{crn} \mathrm{sec}^{-1}$ ) opposing gradients as a function of beam energy, depending upon which of the two alpha distribution functions existed in the plasma. These characteristics become more apparent in Fig. 3, which shows the differential reaction rate as a function of detector angle at various $\mathrm{Li}^{\circ}$ bean velocities for the classical and nonclassical distributions. It is also apparent in this figure that the $\mathrm{He}^{\circ}$ anaiyzer must be located in a forward direction if the signal level is to be maximized. This is a natural consequence of the velocity selectivity of the reaction. Alpha particles are most likely to have low velocities relative to the beam if they are going in somewhat the same direction.

One would also like to be able to discern differences in the distribution function from the results of a single beam energy. That this should be the case is shown by Fig. 4, which gives the velocity distributions of the $\mathrm{He}^{\circ}$ emerging at $20^{\circ}$ (relative to the beam direction) for the (a) classical and (b) nonclassical alpha velocity distributions. These are shown for four different 
$\mathrm{Li}^{\circ}$ injection velocities. We can see that at $\mathrm{Li}^{\circ}$ velocities below $\sim 1.1 \times 10^{9} \mathrm{~cm} \mathrm{sec}^{-1}$ the shapes and centroids of the He $\mathrm{O}^{\mathrm{O}}$ distributions are quite sensitive to the alpha velocity distributions. At $v_{\text {beam }} / v_{0}=0.8$, for instance, the centroid of $\mathrm{He}^{\circ}$ for the classical distribution is shifted down by $\sim 1.7 \times 10^{8}$ $\mathrm{cm} \mathrm{sec}^{-1}$ relative to the injection velocity, while for the nonclassical distribution the centroid of the $\mathrm{He}^{\circ}$ is shifted up by $\sim 1.3 \times 10^{8} \mathrm{~cm} \mathrm{sec}^{-1}$ with respect to the beam energy. In addition, the signal forms resulting from the two assumed distributions differ markedly in shape and width, and, as indicated by Fig. 3, would have somewhat different signal intensities.

Accordingly, we see that this technique yields a number of observables to probe the alpha particle velocity distribution function. At a fixed beam energy, one can measure the shape, intensity, and velocity centroid shift of the emerging $\mathrm{He}^{\circ}$. In addition, with a capability to vary the beam energy, one can measure the rate of change of the $\mathrm{He}^{\circ}$ flux as a function of the $\mathrm{Li}^{\circ}$ velocity.

In order to get an idea of the beam technology required, we need a crude estimate of the expected signal level. If we use the detection geometry assumptions in Ref. 5, then with $Q=1$, an alpha particle density of $10^{12} \mathrm{~cm}^{-3}$, an injected $L i^{\circ}$ beam of 45 $\mathrm{m} A$-equivalent, and subsequent reduction of the beam by one efolding through ionization, we arrive at a He flux of $\sim 8 \times 10^{5}$ $\mathrm{cm}^{-2} \mathrm{sec}^{-1}$ at an analyzer entrance at $20^{\circ}$ relative to the beam direction and located 5 meters from the interaction volume. This is intended as an estimate only; the actual signal flux might be 
somewhat lower due, for instance, to processes such as ionization or charge exchange of the emerging $\mathrm{He}^{\circ}$. This becomes a severe problem for $\mathrm{He}^{\circ}$ at energies below $500 \mathrm{keV}$ (at which energy the loss is about one e-folding). A signal level of the order estimated here should be detectable with relatively conventional techniques similar to those presently employed for charge-exchange analysis of the bulk jon energies in tokamaks.

\section{Required Technology}

The requirement of $\mathrm{a} \mathrm{Li}^{\circ}$ beam of several 10's of milliampsequivalent current at several MeV cannot be met by any existing hardware. However, as we shall briefly discuss, the technological base for development of such a beam appears to exist. A fuller discussion of technological and design considerations of light atom beams for another application (heating) can be found in Ref. 12 .

IV. 1 He Analyzer

Figure 5 shows a conceptual design of an analyzer for the escaping $\mathrm{He}^{\circ}$ resulting from neutralization of the alpha particles in the plasma. At the entrance of the analyzer, a thin foil strips most of the $\mathrm{He}^{\circ}$ back to the alpha particles, which are deflected by a magnet so as to strike detectors. The magnet's purpose is twofold: to momentum analyze the $\mathrm{He}^{++}$, and to allow the detectors to be located behind neutron shielding. If they can be adequately shielded, the most desirable detectors will probably be position-sensitive surface detectors. These would allow energy resolution in addition to that provided by the magnet, and would also permit the rejection of much residual neitron 
noise, since neutrons would generally only lose small amounts of energy in the thin detectors.

\section{2 Source}

The best documented method for producing $\mathrm{Li}^{-}$is a two step process wherein $\mathrm{Li}^{+}$is extracted from an arc source. The positive ions then pass through a metal vapor where a fraction of them acquires two electrons to become negative ions. The most developed approach to high current production of $\mathrm{Li}^{+}$is with the calutron type sources of Oak Ridge National Laboratory. These have a long history, and have yielded $\mathrm{Li}^{+}$current densities of as much as $72 \mathrm{~mA} / \mathrm{cm}^{2}[13]$, and total $\mathrm{Li}^{+}$currents as large as 1.2 amps [14] while operating continuously. Some measurements have been made of positive-to-negative ion conversion efficiencies with small beams. Approximately 98 of a $5 \mathrm{keV} \mathrm{Li}^{+}$heam was converted to $\mathrm{Li}^{-}$while passing through a cesium vapor cell [15]. Thus a source to produce $\sim 100 \mathrm{~mA}$ of $\mathrm{Li}^{-}$might consist of a $\mathrm{Li}^{+}$ source using calutron technology and extracting the beam in an accel-decel mode, followed by a transverse supersonic cesium jet. There are at least two other possible alternative approaches to a high current $\mathrm{Ii}^{-}$source. One possibility is to produce the negative ions directly with a surface plasma source similar to one of the types being developed for $D^{-}$production $[16,17,18]$. However, while such sources are very attractive for making negative ions from gaseous feedstocks, they may be less suitable for producing negative ions of a substance, such as lithium, which has a lower vapor pressure than does the cesium which dopes the converter 
surface. Another method which might be highly desirable, but which is unexplored at this time, is photodissociation of NaLi molecules in a molecular beam into $\mathrm{Na}^{+}$and $\mathrm{Li}^{-}$using 2350-2400 $\mathrm{A}$ photons from a laser [19].

IV. 3 Accelerator

Accelerating $100 \mathrm{~mA}$ to energies as high as $5.3 \mathrm{MeV}$ would be an intractable problem for a conventional electrostatic accelerator. A RF linear accelerator with electric quadrupole focusing $[20,21]$ is a much more suitable choice, since the electrical potentials with respect to ground that are required can be much lower than the final beam energy, and the velocity-independent electric quadrupole focusing allows one to input a low velocity beam with high space charge density. RF quadrupole (RFQ) accelerators [20], which are being developed at Los Alamos Scientific Laboratory, seem to be especially appropriate for this application. They appear to be capable of accelerating the full $100 \mathrm{~mA}$ current in a single accelerator channel, they can have a large entrance aperture to accept the $\mathrm{Li}^{-}$beam, and they can readily be pumped through grifided ducts. A preliminary design of a RFQ to accelerate $100 \mathrm{~mA}$ of $\mathrm{Li}^{-}$ to $6 \mathrm{MeV}$ has been done by LASL [20]. In the design of Ref. 20, the input aperture radius would be large $(8.47 \mathrm{~cm}$, of which about half could be filled with beam), and the output beam would have a radius of asout a centimeter. The normalized emmitance acceptance would be $\sim .21 \mathrm{~cm}$ mrad [22]. This should be adequate to use with the two stage $\mathrm{Li}^{-}$source, but the acceptance could probably be further increased if the source optics required it [22]. Using 
$50 \mathrm{MHz}$ RF at $223 \mathrm{kV}$, the length would be asout 7 meters. The $\mathrm{Li}^{-}$ would be electrostatically preaccelerated to $100 \mathrm{keV}$ prior to injection into the RFQ. The capture and transmission efficiency of a RFQ can be high ( $93 \%$ for this case, in the absence of premature stripping 1osses), which is desirable since the $\mathrm{Li}^{-}$is not easy to make.

Although much information abo: - the alpha particle velocity distribution function can be obtained by injecting the $\mathrm{Li}^{\circ}$ beam at a single energy, it is clearly desirable to have the capability to inject at any of several discrete energies (continuous variability, while useful, is not required). Changing the energy of a RF accelerator is not as straightforward as would be the case with an electrostatic accelerator. However, Ref. 20 has discussed methods of incorporating energy variability which should be adequate for this application. These possible methods include following the RFQ with independently phased cavities, varying the frequency, or varying the drive voltages to sections of the RFQ.

\section{TV. 4 Neutralizer}

Unlike heavier negative ions, $\mathrm{Li}^{-}$can be neutralized quite efficiently by passage through a thin gas cell. At $6-7 \mathrm{MeV}$, a nitrogen cell has been oiserved to neutralize $\sim 458$ of a $\mathrm{Li}^{-}$beam $[23,24]$, and a hydrogen gas cell can give a neutral yield of $54 \%$ [23]. Since the ritrogen can be kept at a much lower vapor pressure than can hydrogen by cryocondensation pumping, and since we would like to minimize the gas load to the accelerator in order to suppress premature stripping, we would choose nitrogen rather than 
hydrogen as the neutralizer gas. The neutralization efficiericy obtainable with a gar cell is adequate for this diagnostic application, where power efficiency is not of paramount importance. However, if photodetachment neutralizers were developed for $\mathrm{D}^{-}$heating beams, then one could be adapted for use with $\mathrm{Li}^{-}$ to yield a higher neutral fraction. The photodetachment cross section for $\mathrm{Ii}^{-}$has been calculated [25] to be grossly similar in shape to that of $\mathrm{D}^{-}$, but larger. Accordingly, any photodetachment system for $\mathrm{D}^{-}$should also be effective with $\mathrm{Li}^{-}$.

\section{5 Beam System}

Figure 6 shows a conceptual design of a $\mathrm{Li}^{\mathrm{O}}$ beam incorporating a two-stage $\mathrm{Li}^{-}$source and a RFQ accelerator. The analyzing maynet after the source section allows drifting metal vapor to be condensed before the accelerator. The deflection magnet after the RFQ permits the accelerator to be shielded srom the direct neutron flux of the tokamaik. Focusing magnetic quadrupoles would probably be necessary prior to the neutralizer. A gas neutralizer would necessarily be followed by a deflectjon magnet (probably air core) and cooled dumps for the residual ions. These ions, both positive and negative, could carry as much as $300 \mathrm{KW}$. Fortunately, at the gas cell thickness which yields the maximum neutral fraction, the power in the unneutralized remnant is about equally aivided between the positive and the negative ions [24], so the power density on the dumps is decreased. A mure comprehensive discussion of system aspects of light atom beams is contained in Ref. 12 .

V. Conclusion

Our calculations indicate that this technique can yield useful 
information about the velocity distribution function of the fast confined alpha particles resulting from $D-T$ fusion reactions. It arpears that $\mathrm{Li}^{\circ}$ is the most desirable doping beam to use, witr. a charge-exchange analyzer to measure the escaping ife $^{0}$ which results. The $100 \mathrm{~mA} \mathrm{Ii}^{-}$beam at several MeV wich is required for this diagnostic will need significant development, but from our siudy it does appear possible based upon our present state of knowledge. There are some atomic physics questions requiring further theoretical examination-namely excitation of $\mathrm{Li}^{\circ}$, the double charge-exchange cross seciions for any significant excited states of $\mathrm{Li}^{\circ}$, and the distribution and fate of excited states of helium resulting from double charge exchange. Nonetheless, neutralization of the fast alphas by an energetic neutral lithium bearc seems to hold promise as a viable and feasible measurement technique.

\section{Acknow ledgments}

For the use of unpublished data we thank R. W. Mcculiough, T. V. Goffe, M. Lennon, M. B. Shah, and H. B. Gilbody of the Queen's University of Belfast, and G. A. Murray, J. Stone, M. Mayo, and T. J. Morgan of Wesleyan University. It is also a pleasure to thank G. D. Alton, W. A. Bell, K. H. Berkner, J. Clarke, K. W. Ehlers, H. P. Eubank, J. R. Hiskes, E. B. Hooper, Jr., K. N. Leung, I. O. Love, A. W. Maschke, R. V. Pyle, A. S. Schlacter, J. A. Schmidt, L. D. Stewart, R. H. Stokes, D. A. Swensen, and T. P. Wangler for helpful discussions, and in particular J, C. Weisheit for his work on $\mathrm{He}^{-}$atom physics. 
FIGURE CAPTIONS

1. Double charge-exchange cross section for $\mathrm{He}^{++}+\mathrm{Li}^{\circ} \longrightarrow \mathrm{He}^{\mathrm{O}}$ $+\mathrm{Ii}^{++}$from References 10 and 11 . The curve has been extrapolated below $28 \mathrm{keV}$ and above $800 \mathrm{keV}$.

2. Values of $\langle\sigma v\rangle$ for the (a) classical and (b) nonclassical alpha particle velocity distribution functions.

3. Differential $\langle\sigma v\rangle$ for the (a) classical and (b) nonclassical alpha particle velocity distributions shown as a function of the angle between the neutral particle detector and the doping beam for several beam velocities. $v_{0}$ is the alpha birth velocity of $1.3 \times 10^{9} \mathrm{~cm} \mathrm{sec}^{-1}$.

4. Velocity distributions (normalized to the same peak amplitude) of the emerging $\mathrm{He}^{\circ}$ at $20^{\circ}$ as a function of the $\mathrm{Li}^{\circ}$ beam velocity for the (a) cl.assical and (b) nonclassical particle velocity distributions. The arrows indicate the beam injection velocities at $v_{\text {beam }} / v_{0}=0.4,0.6,0.8$, and 1.0 , where $v_{0}$ is the alpha particle birth velocity.

5. Conceptual design of a neutral helium analyzer. The stripped helium ions are masnetically deflected to effect momentum a:ialysis and shielding of the detectors.

6. Conceptual design (not to scale) of a beamline to produce multi-MeV Li ${ }^{\circ}$. 
REFERENCES

[1] Ya. I. Kolesnichenko, "The Role of Alpha Particles in Tokamak Reactors," Nucl. Fusion 20, 727 (1980), and references contained therein.

[2] L. M. Hively, G. H. Miley, "Fusion Product Bombardment of a Tokamak First Wall," Nucl. Fusion 17, 1031 (1977).

[3] L. M. Hively, G. H. Miley, "Prompt and Non-Prompt Contributions to Fusic. Product Bombardment of a Tokamak First Wall," Nucl. Fusion 20,969 (1980).

[4] R. J. Goldston, "Charge-Exchange Spectra Near the Injection Energy in Tokamaks Equipped with Tangential Neutral Beams," Nucl. Flision 15, 651 (1975).

[5] D. E. Post, D. R. Mikkelsen, R. A. Hulse, L. D. Stewart, J. C. Weisheit, "Techniques for Measurirg the Alpha Particle Distribution in Magnetically Confined Plasmas," Journal of Fusion Energy 1, 129 (1981)。

[6] E. B. Hooper, Jr., P. A. Pinosy, P. Poulsen, C. F. Burrell, L. R. Grishäm, and D. F. Post, "High current Source of $\mathrm{He}^{-}$ Ions," Rev. Sci. Instrum. 51, 1066 (1980).

[7] K. W. Ehlers and K. N. Leung, "He- Ion Froduction for Alpha Particle Distribution Measurements in Tokamaks," Bull. Am. Phys. Soc. 25, $698(1980)$.

[8] A. S. Schlacter, "Metastable Contamination of Fast He ${ }^{\circ}$ Beams Produced from He ${ }^{-}, "$ Bull. Am. Phys. Soc. 25, 698 (1980).

[9] L. R. Grisham, D. E. Post, J. C. Weisheit, H. P. Eubank, D. R. Mikkelsen, L. D. Stewart, "Status Report of the Fusion Alpha Confinement Test March 1980," Princeton University 
Plasma Physics Laboratory Report. PPPL - 1661 (1980).

[10] R. W. McCullough, T. V. Goffe, M. Lennon, M. B. Shah, H. B. Gilbody, "Electron Capture by $\mathrm{He}^{+}$and $\mathrm{He}^{2+}$ Ions in Li Vapor," Abstracts of XII International Conference on the Physics of Electronic and Atomic Collisions, Sheldon Datz, Ed., Gatlinburg, Tenn., July 15-21, 1981, Vol. 2, 561 (1981).

[11] G. A. Murray, J. Stone, M. Mayo, and F. J. Morgan, "Single and Double Electron Transfer in $\mathrm{He}^{2+}+\mathrm{Li}$ Collisions," to be published in phys. Ref. A.

[12] L. R. Grisham, D. E. Post, D. R. Mikkelsen, H. P. Eubank, "Plasma Heating with Multi-MeV Neutral Atom Beams," Nuclear Technology/Fusion 2, (April 1982).

[13] "Aspects of the physics, Chemistry and Technology of High Intensity Heavy Ion Sources," G. D. Alton, Nuclear Instruments and Methods 189, 15-42 (1981).

[14] L. O. Love, R. N. Goslin, W. A. Bell, H. B. Greene, and K. W. Prater, "Investigation of the Possibilities of Lithium Isotope Collection Using Alpha-2 Equipment," Oak Ridge National Laboratory Report ORNL-Y-728 (1951).

[15] P. Tykesson, "The Production of Negative Heavy Ion Beams through Charge-Exchange Process," Symposium of Northeastern Accelerator Personinel, Oak Ridge, Tennessee, October 23-25, 1978 .

[16] K. W. Ehlers and K. N. Leung, "Multicusp Negative Ion Source," Rev. Sci. Instrum. 5I, 721 (1980). 
[17] W. K. Dagenhart, W. L. Stirling, H. H. Haselton, G. G. Kelley, J. Kim, C. C. Tsai, and J. H. Whealton, Modified Calutron Negative Ion Source Operation and Future Plans," Proceedings of the Second International Symposium on the Production and Neutralization of Negative Hydrogen Ions and Beams, Brookhaven, New York, October 1980, Th. Sluyters, Ed., ENL 51304, 217 (1981).

[18] K. Prelec, "Progress in the Development of High Current SteadyState $\mathrm{H}^{-} / \mathrm{D}^{-}$Sources at $\mathrm{BNL}, "$ Proceedings of the second International Symposium on the Production and Neutralization of Negative Hydrogen Ions and Beams, Brookhaven, New York, October 1980, Th. Sluyters, Ed., BNL 51304, 145 (1281).

[19] C. W. Jtwalley, University of Iowa, private communication (1980).

[20] T. P. Wangler and R. H. Stokes, "The Radio Frequency Quadrupole Linear Accelerator," IEEE Transactions cr Nuclear s.iience NS-28, 1494 (1981).

[21] R. M. Mobley, J. J. Brodonski, G. M. Gammel, J. T. Keane, A. W. Maschke, and R. T. Sanders, "MEQALAC (Multiple Electrostatic Quadrupole Linac): A New Approach to Low Beta RF Acceleration," IEEE Transactions on Nuclear Science NS-28, $1500(1981)$.

[22] T. P. Wangler, Los Alamos Scientific Laboratory, private communication (1981).

[23] J. P. Aldridge and J. D. King, "Charge-Exchange Cross-Sections for $\mathrm{Li}^{-}$Ions at $6 \mathrm{MeV}, "$ Los Alamos Scientific Laboratory Report LA-8682-MS (1981).

[24] L. R. Grisham, D. E. Post, B. M. Johnson, K. W. Jones, J. Barrette, T. H. Kruse, I. Tserruya and Wang Da-Hai, 
"Efficiencies of Gas Neutralizers for Multi-MeV Beams of Light Negative Ions," Review of Scientitic Iistrumtents 53(3) (March 1982).

[25] D. W. Norcross and D. L. Moores, "Photodetachment of $\mathrm{Li}^{-}$ and $\mathrm{Na}^{-}, "$ Atomic Physics 3: proceedings of the Third International Conference on Atomic Physics, August 1972, Boulder, Colorado, S. J. Smith and G. K. Walter, Eds., Plenum Press, New York - London, p. 261-267 (1973). 
\#81 X1327

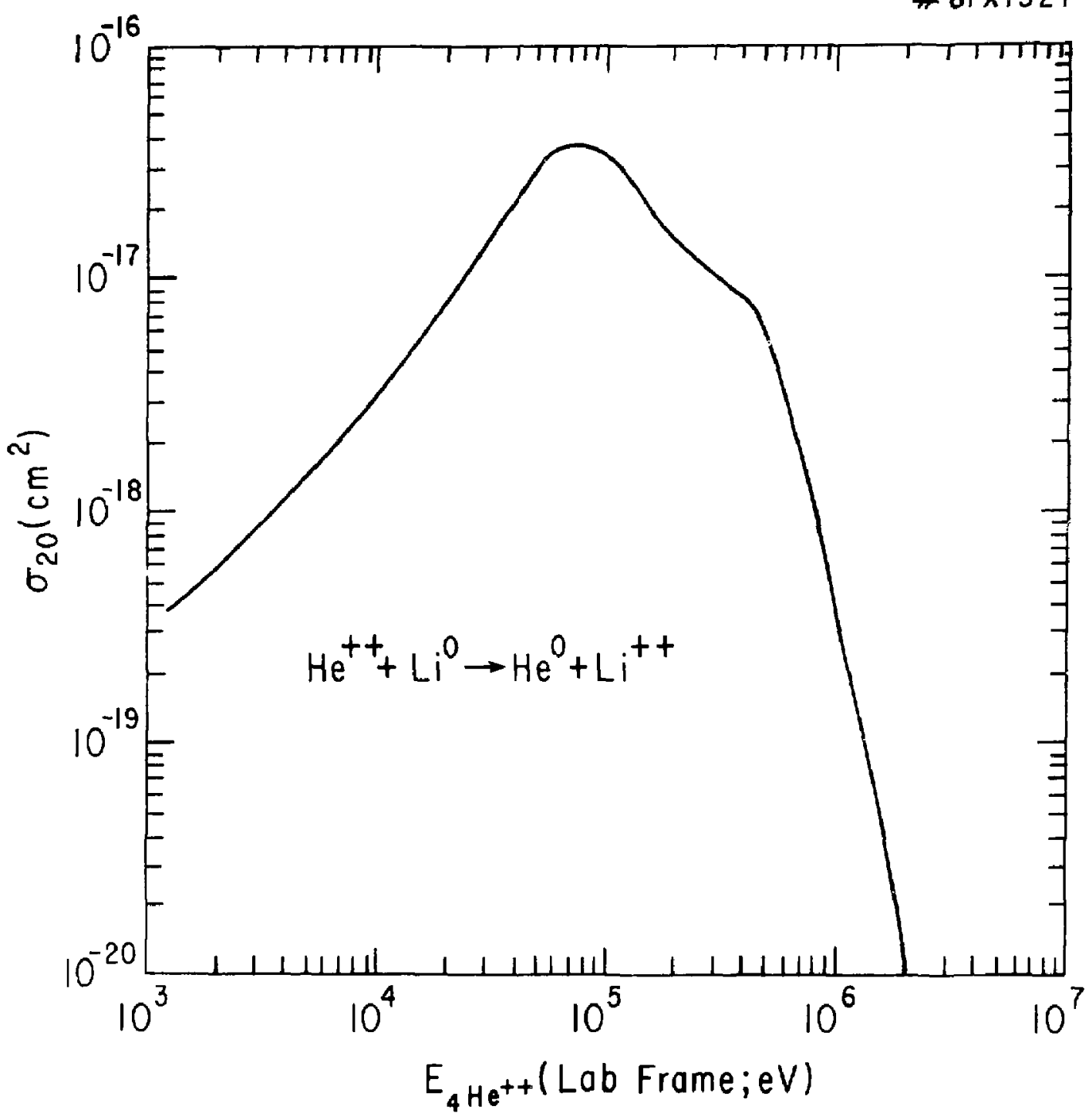

FIE. 1 


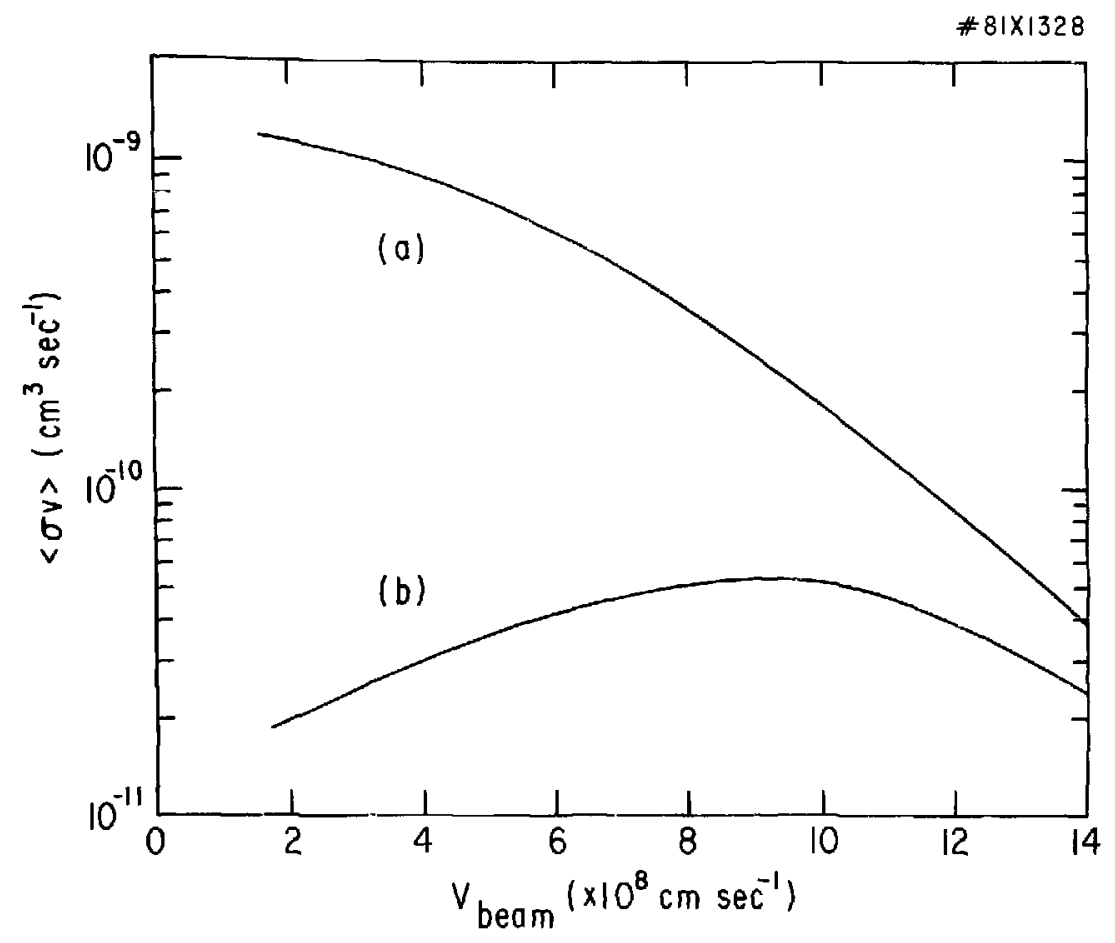



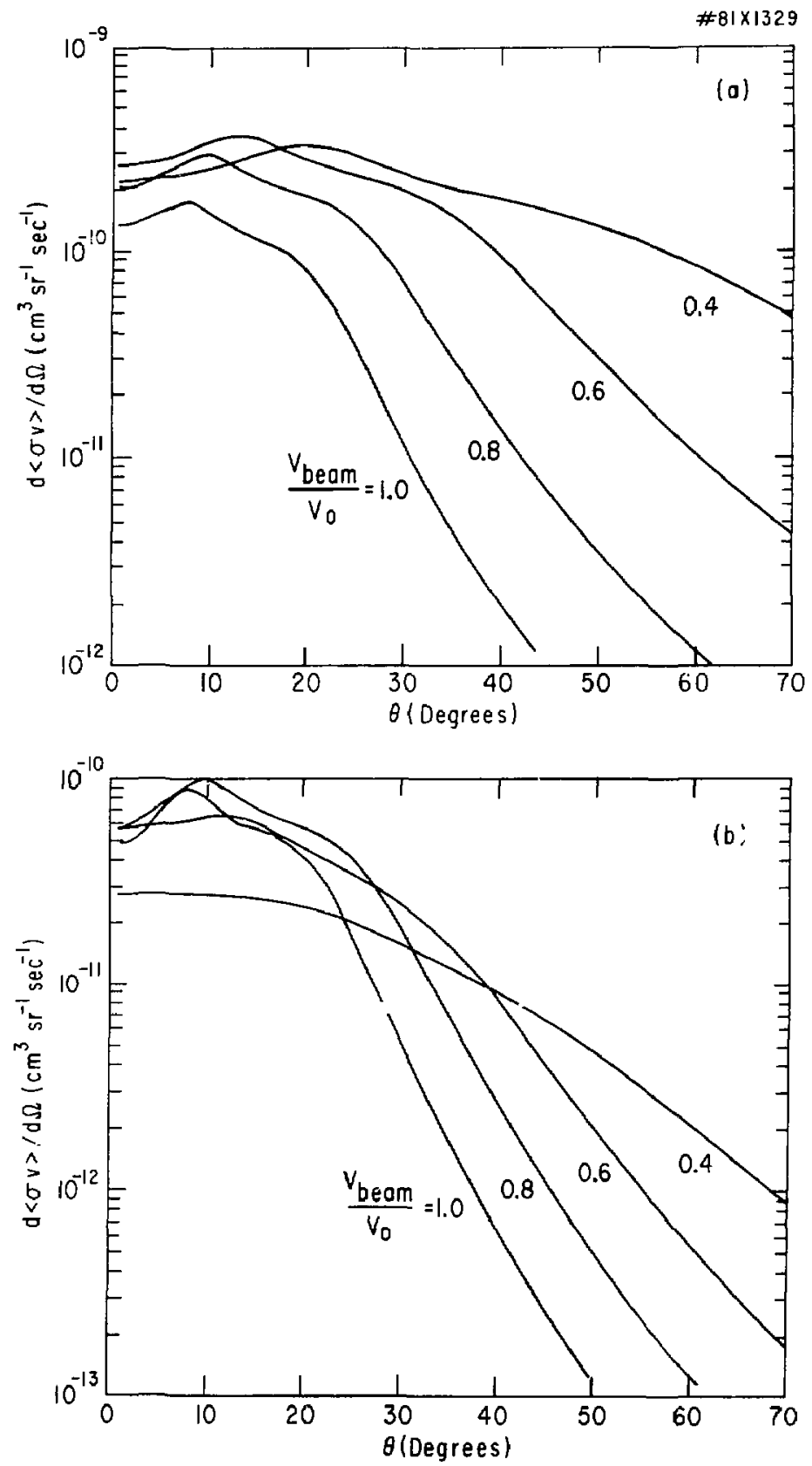

FIG. 3 
PARTICLE FLUX (Renormaiized To Equa! Peaks)
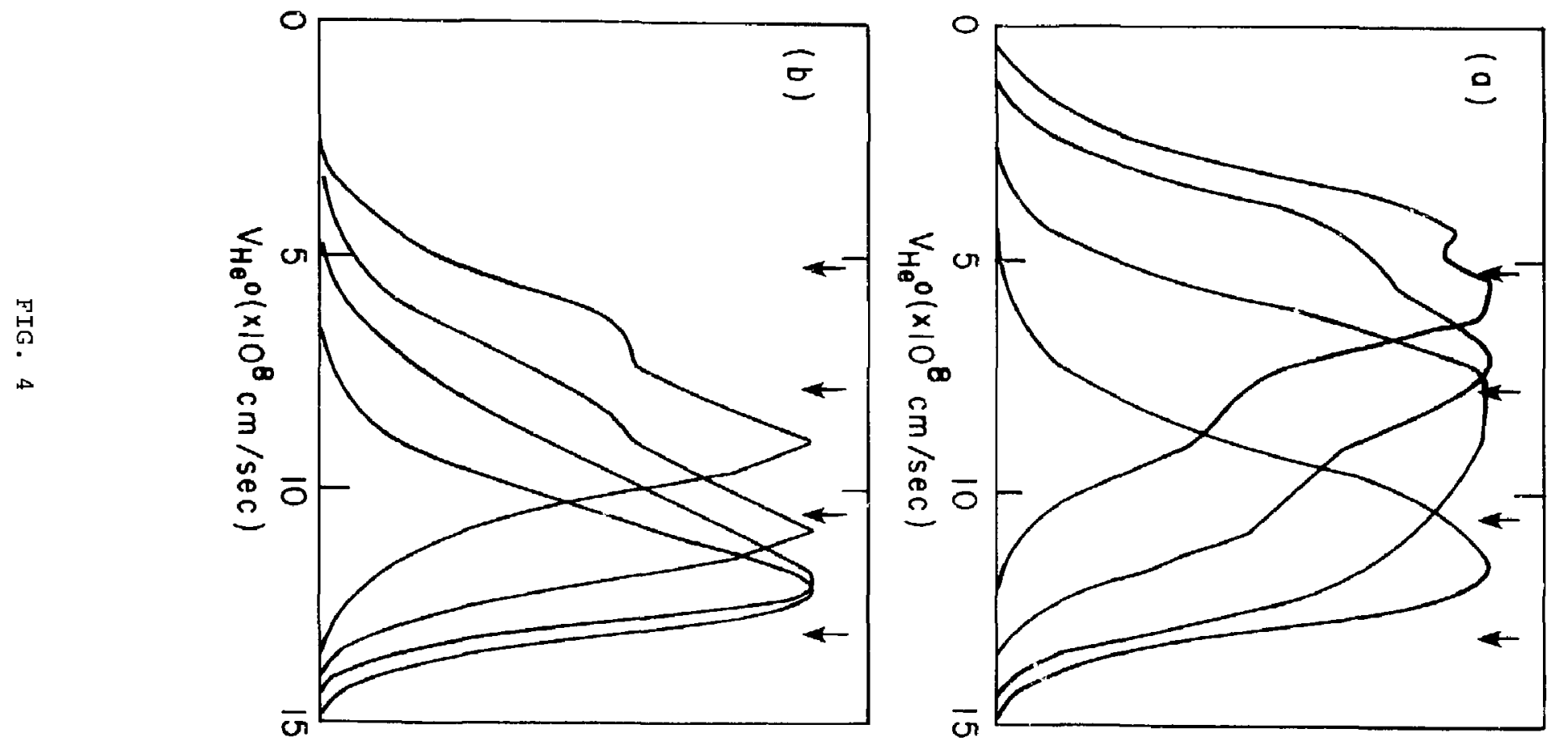


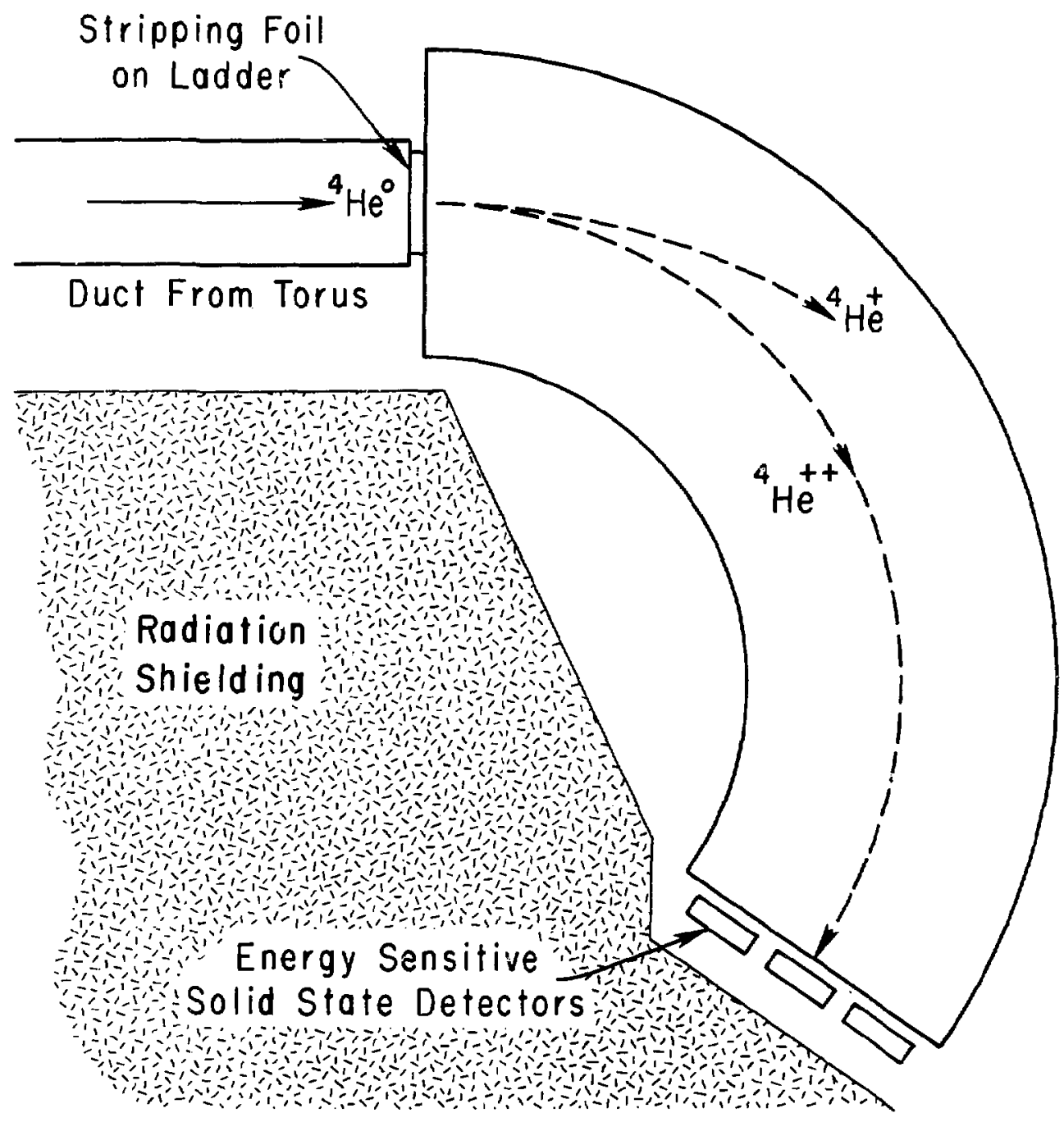

FIG. 5 
$\mathrm{Li}^{-}$BEAMLINE USING A TWO-STAGE SOURGE

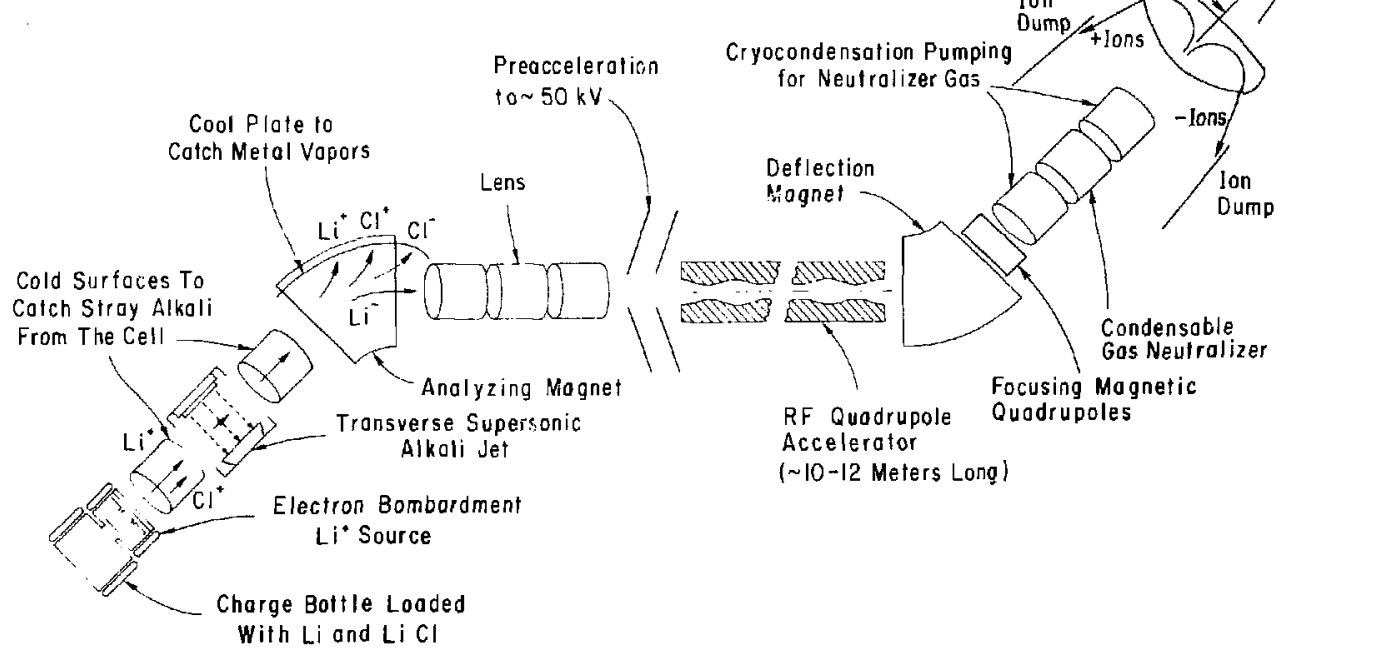

